\title{
Constructing Less Congested Route for Data Transmission by Selecting High Energy Nodes among Cost Efficient Trusted Neighbors for Improving QOS in WSN
}

\author{
Lakshit Bakshi, Dinesh Arora \\ (ECE department, SDDIET, Kurukshetra University, India) \\ (Prof. and Dean GVIET, Banur, Rajpura, P.T.U, India)
}

\begin{abstract}
Sensor networks are strong proposal in almost every wireless application. Secure and reliable communication without expense of throughput is always a key concerned. Although trust based cost efficient scheme have already employed but use of same congested route leads to degradation of quality of service due to frequent usage of trusted congested route. Our research work is based on a fact that high energy node are always stable and less prone to congestion because of their less usability in routing path. Propose article presents applicability of energy level on cost efficient trusted neighbors to select high energy nodes to construct less congested route for data transmission to provide throughput enhancement and improving quality of service along with secure communication.
\end{abstract}

Keywords: QoS, Sensor network, Throughput.

\section{Introduction}

Recent developments occur in various fields like information science, medicine, military and many more due to the advancement of wireless technology in last few years. Additionally with the 3rd generation radio technology, high quality multimedia services (voice, video $\&$ data) over wireless networks have become a reality. [1]. Wireless sensor networks (or WSNs) consist of thousands of sensor nodes, which is capable of sensing and computing the events in wireless communication systems. These nodes or sensors processes the events happening near sensor by sensing electronic conditions related to the environment surrounding the sensor and transduce them into electrical signals. Data transmission occurs from transmitting node to sink node, which communicate each other via large number of intermediate nodes or directly to an external base station

\section{Formulating A Problem}

Wireless sensor networks consist of spatially distributed sensors to monitor physical or environmental area. Wireless sensor networks are made of nodes from few to several hundred nodes, where each node is connected to a sensor. Data is transmitted through node-to-node where distance determination is necessary. Due to large distance, energy is more utilized. In earlier systems only distance is considered and even few enhancements have been made by experts had also gone through energy cost and surety level of nodes like TRAF to avoid attack of malicious nodes. These are called trusted nodes. ). Such a scheme although provide high packet delivery rate and less transmission delay but no stress is given on throughput enhancement as it provide all neighbor-trusted nodes with energy cost require for transmitting a packet but no emphasis is given on nodes with same value of energy cost; so continuous transmission over these nodes leads to congestion over data route and affects the throughput.

\section{Problem Solution}

Proposed work is different in the sense that each trusted neighbor node along with energy cost is labeled with energy value as well. Among all neighbor-trusted nodes with equal value of energy cost, node with high-energy value is preferred over low energy node. Nodes are labeled as 1,2or3 with one being highest energy value of node and 3 being lowest energy value of node. High-energy nodes are preferred because they are more stable and less likely to take part in routing path and hence less congestion occur on those nodes. In proposed work, data routing scheme is employed which not only consider distance and surety level of nodes but main emphasis is also done regarding energy level of each node with the application of energy level table in the routing table of AODV thereby enhancing quality of service to provide secure as well as effective communication in wireless sensor networks

\section{Work Methodology}

- The very first step is to initiate the parameters of the networks such as

1) How much area is covered under the network? 
2) What is the frequency of transmission required?

3) What kind of data is to be transmitted?

4) Numbers of nodes etc.

- Detecting total number of nodes in the network area by connecting a sensor to each of them. We have presented an algorithm to detect the compromised nodes in wireless sensor networks. These nodes are capable of performing tasks some processing, gathering sensory information and communicating with other connected nodes in the network.

- Now these nodes are initialized, which means to provide initial values before communication according to their location in the network. This initial value corresponds to their characteristics.

- According to our module calculate the total numbers of nodes associated to other node in the network, which means to have a information about which node is neighbor to other one. It even gives information about how many nodes are associated to each node.

- Now define the source and sink in the networks from calculated numbers of nodes to start the data transmission.

- A static data table with node distance, energy level and surety level is obtained from observed data. The source node will now find the shortest path for communication, which is based on the data calculated. The node with least distance from source with highest energy level and surety with minimum energy cost will be transmitted data from source. Thus, an acknowledgement will be send back to the source informing that whether data received or lost. If the acknowledgement received is positive means, data received and if negative means data is lost.

- After receiving data from source this node will act as source and send data to other neighboring shortest distant, highest energized and high sureness node. The static table always updated after every successive transmission of data.

- At last coding function is given to a network simulator i.e. NS2 SIMULATOR, which is used for data transmission. This module leads to less energy is consumption and even Failure of communication is reduced.

\section{Experimental Setup}

The performance of the proposed algorithm is analyzed by initializing some network parameters before actual coding. In proposed algorithm, network with specific number of nodes (which are 15 in our case) is distributed within an area of $600 * 600$ (in meters). Nodes are deployed at a specific location assigned to then on $\mathrm{x}$-axis and $\mathrm{y}$-axis as shown in figure below

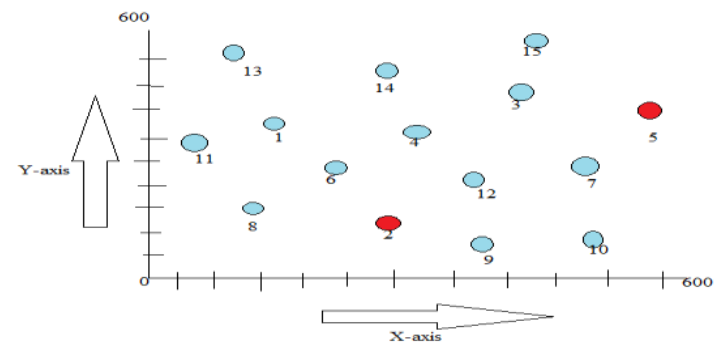

Figure 1: Deployment of Sensor Nodes

After nodes deployment in sensor field, system is assigned with some predefined value of parameters such as initial energy associated with each node, type of antenna used within each node, maximum simulation time etc. These are called simulation parameters. Table below summarizes the simulation parameters used.

Table 1: Simulation Statistic

\begin{tabular}{|l|l|}
\hline Name of the parameter & Associated value \\
\hline Maximum simulation time (in second) & 10 \\
\hline Area size & $600 * 600$ \\
\hline Number of nodes used in setup & 15 \\
\hline Initial energy (in joules) & 100.00 \\
\hline Maximum packets in queue & 500 \\
\hline Traffic type & CBR (Constant Bit Rate) \\
\hline Routing protocol used & AODV \\
\hline Antenna type & Omni directional \\
\hline Type of channel & Wireless \\
\hline Queue type & Drop tail \\
\hline Propagation Type & Two Ray Ground \\
\hline Protocol Type & IEEE 802.11 MAC Protocol \\
\hline
\end{tabular}




\section{Experimental Results}

This section incorporates performance of the generated system model graphically in terms of performance evaluating parameters.

Throughput v/s Initial Energy: Performance of the proposed system can measured in terms of throughput, which can defined as number of packets received correctly at sink node over a unit period of time (usually a second). Throughput is measure in kbps. Throughput can also measured as ratio of number of correctly delivered data packets at sink node to the number of all data packets transmitted.

Simulation results were obtained; graph below indicates throughput versus initial energy of the system

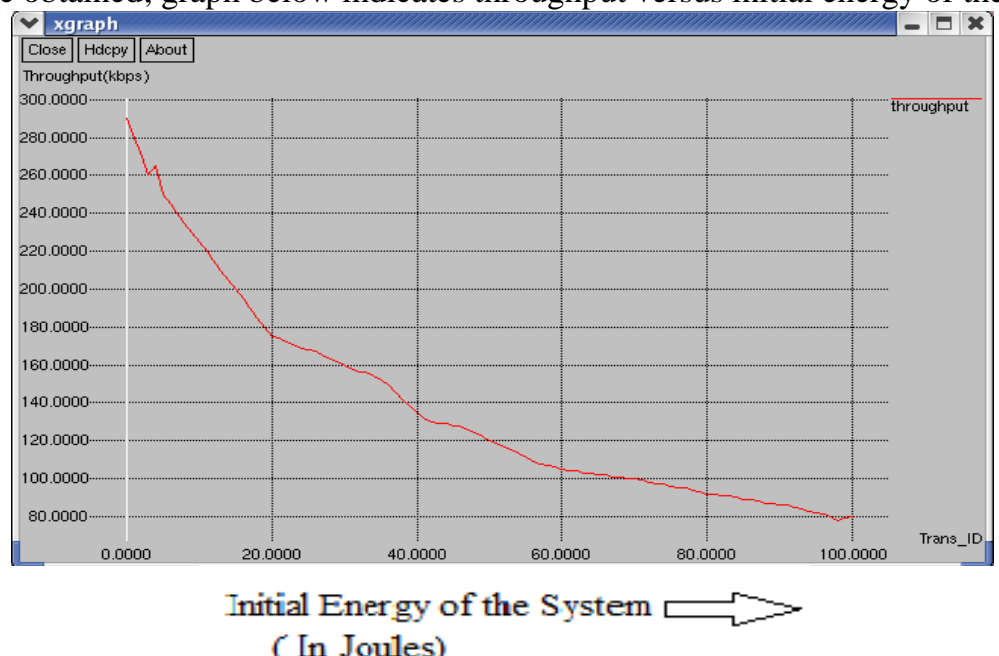

Figure 2: Throughput v/s Initial Energy of the System

As we know, at the start of simulation, initial energy of the system is kept $100 \mathrm{~J}$ in simulation statistic table. Therefore at time $\mathrm{t}=0$ when energy of the system is $100 \mathrm{~J}$, system is about to start transmit packet and at that time as no packet is received at destination nodes and hence the throughput is less. As the system start simulating and energy of system start to drop, packets start receiving at destination nodes thereby increasing throughput of the system

Lesser the number of nodes participate in the routing path, more is the chance that data packets received correctly at destination and hence more the throughput. Therefore, for particular set of source and destination node, throughput may vary according to the number of nodes participating in routing process.

Packet Drop Rate v/s Node Density: Performance of the proposed system can be evaluated in terms of packet drop ratio. Drop rate can be defined as ratio of number of packets dropped to the total number of packets received by a node. Mathematically,

Drop rate $(\%)=$ Number of packets dropped $\quad x 100$

Total number of packets received

Simulation results were obtained, graph below indicate packet drop rate versus node density of the network.

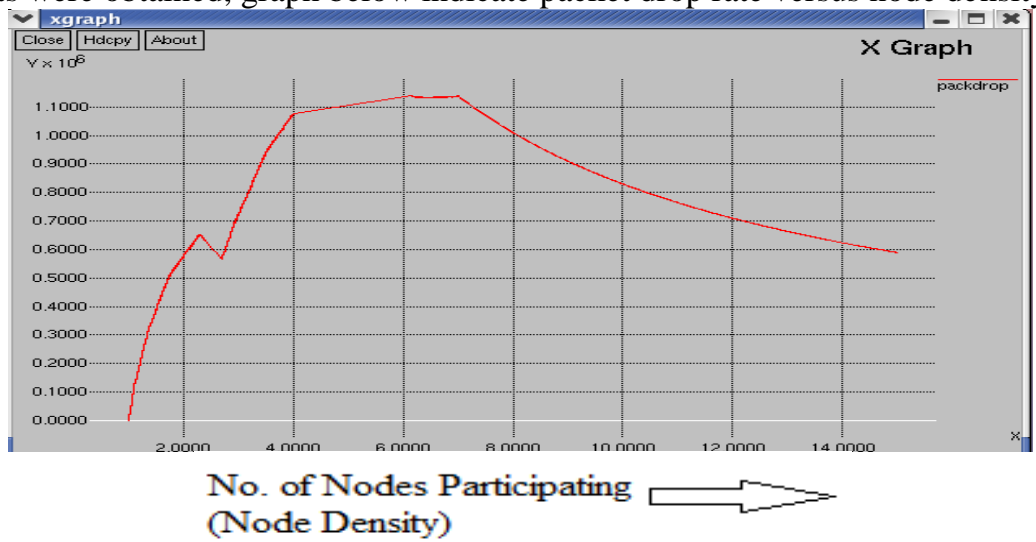

Figure 5: PDR v/s Node Density

Initially at simulation time $\mathrm{t}=0$, when no node is participating in the routing process; obviously there is no packet transmission and hence packet drop is 0 ; this can be visualized in the graph. As the node density increases and more nodes starts to participate in the routing process network is now more venerable to attack by untrusted nodes and hence more chance of packet to loss thereby increasing packet drop rate. When more number of nodes start participating, probability of alternate path selection increases thereby avoiding 
incorporation of untrusted nodes in the route hence probability of packet drop decreases eventually in the later half. Hence proposed scenario offers less packet drop rate when network size increase.

Transmission delay v/s Number of Hops in Transmission: Delay of individual packet is the difference between times a packet takes to reach the final destination node from originating time of a packet from source node. Therefore, transmission delay (or end-to-end delay) is the ratio of sum of all such delays of each packet to the number of packets transmitted from source to destination.

Packet Delay $=($ Packet Reach Time at Destination $)-($ Originating Time of Packet $\quad$ from source $)$ Transmission Delay $=$ Sun of Delay of all Packets $/$ Total Packets Transmitted.

Simulation results obtained, graph below depicts transmission delay from source node to destination versus number of hops (total round) a packet take to reach until destination node encounters.

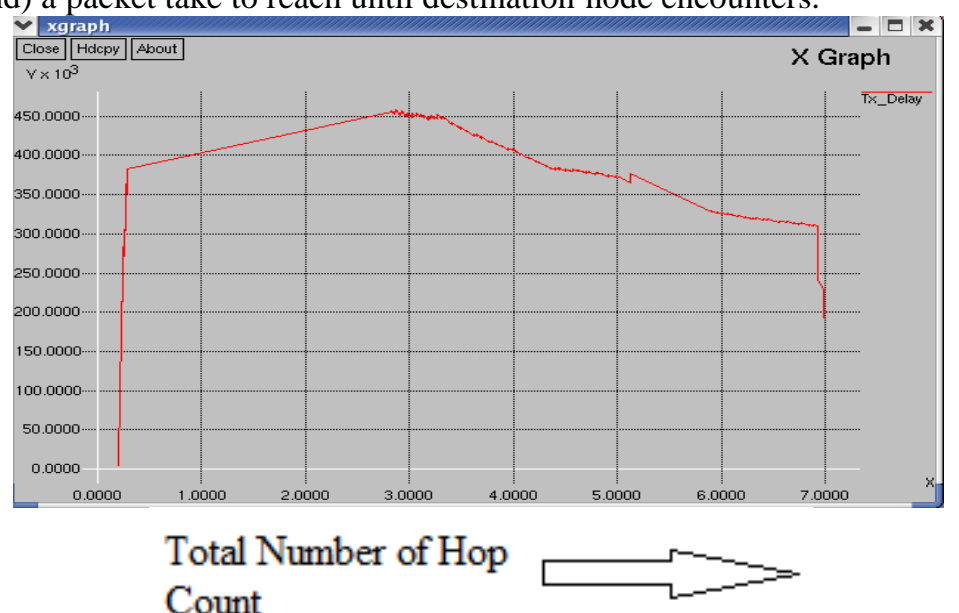

Figure 6: Transmission Delay v/s Hop Count

Initially at start of simulation when packet is at source node with hop count $=0$; no delay is observed as packet is still to be transmitted. As the intermediate nodes (hop count) in the routing path of packet increases, packet takes longer time to reach destination node and hence delay increases. Proposed algorithm selects intermediate nodes with high trust value along with high energy value so that delay at each node occurs less. So with further increase in hope count gradually decrease packet delay time to reach until destination and hence decreases transmission delay.

\section{Result Validation}

Throughput of the proposed system when simulated and compared with existing system shows a satisfactory progress. Figure below compares the throughput of the proposed and existing system.

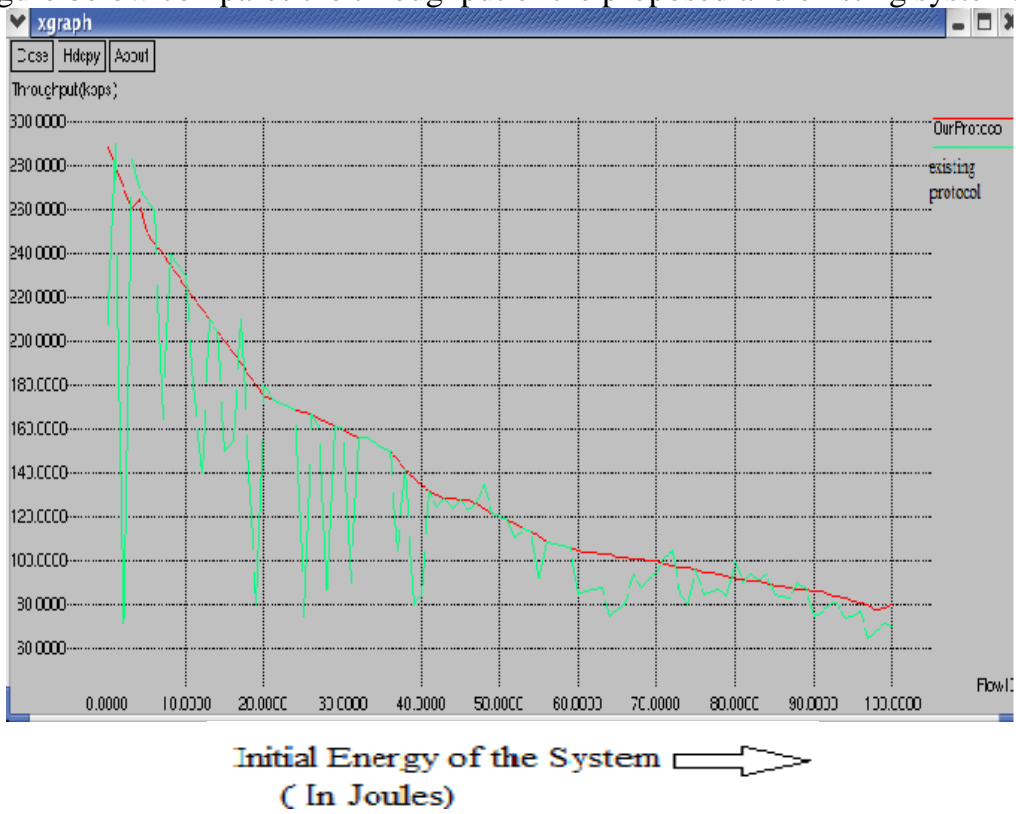

(In Joules)

Figure 7: Comparison of Throughput 
Above graph defines the throughput of existing protocol and proposed protocol against initial energy of the system. Simulation was running in 10 seconds of time. It is clear from the graph as initial energy of the system starts to decrease, throughput of the existing system keep on oscillating between lower and higher values but the throughput of the proposed system always remain near to peak value of the existing system. This is obvious due to the fact of introducing the energy cost per hop and energy level of each trusted-neighbor node which not only avoid malicious node but also enhances number of packet delivery per unit of time

\section{References}

[1] Pooja Kalidas Shinde, Veeresh Gangappa Kasabegoudar "Energy efficient and trust metric based routing technique using collection tree protocol for WSNs" International Journal of Sensors and Sensor Networks, 2013; 1(5): 61-68 Published online September 30, 2013.

[2] Theodore S. Rappaport, "Wireless Communications: Principles and Practices (2nd edition)," Prentice Hall, 2002

[3] Sridhar Subramaniana, Baskaran Ramachandran "Trust Based Scheme for QoS Assurance in Mobile Ad-Hoc Networks"

[4] Guoxing Zhan, Weisong Shi, and Julia Deng "TARF: A Trust-Aware Routing Framework for Wireless Sensor Networks"

[5] Rajiv K. Nekkanti, Chung-wei Lee "Trust Based Adaptive On Demand Ad Hoc Routing Protocol” 